\title{
Novel fabrication of silica nanotubes using multi-walled carbon nanotubes as template
}

\author{
ZHI-HUA YIN, XIANG LIU and ZHONG-XING SU* \\ College of Chemistry and Chemical Engineering, Lanzhou University, Lanzhou 730000, PR China
}

MS received 30 November 2008

\begin{abstract}
Silica nanotubes were synthesized using multi-walled carbon nanotubes (MWCNTs) as template. The as-obtained samples were characterized by infrared spectroscopy (FTIR), X-ray diffraction (XRD), transmission electron microscopy (TEM), field emission scanning electron microscope (FE-SEM) and photoluminescent (PL) spectroscopy. The results indicate that the thickness of the outer walls is about $10 \mathrm{~nm}$ and the inner diameter is completely dependent on the size of MWCNTs. The as-fabricated silica nanotubes emit a strong violet light under excitation of $250 \mathrm{~nm}$.
\end{abstract}

Keywords. Silica nanotubes; template; functionalization; MWCNTs.

\section{Introduction}

In the past few decades, one-dimensional (1D) nanostructure materials (nanotubes, nanowires, nanobelts and nanorods) were extensively studied due to their novel physical and chemical properties (Feldman et al 1995; Alivisatos 1996; Ye et al 2002; Zhang et al 2007), especially with respect to the promising applications in numerous areas such as nanoscale electronics and photonics. Earlier efforts were mainly devoted to the research of carbon nanotubes, metals and II-VI semiconductors (Schonenberger et al 1997; Zhang et al 2000). Recently inorganic nanotubes derived from non-layered structures such as $\mathrm{SiO}_{2}, \mathrm{Al}_{2} \mathrm{O}_{3}, \mathrm{TiO}_{2}, \mathrm{Ga}_{2} \mathrm{O}_{3}, \mathrm{CdSe}, \mathrm{CdS}$, $\mathrm{ZnS}$ have attracted intense interest for their excellent performance (Nakamura and Matsui 1995; Hoyer 1996; Cheng and Samulski 2001; Xiang et al 2003; Golberg et al 2005; Guo et al 2005; Zollfrank et al 2007). Compared with other tubular nanomaterials, pure silica nanotubes not only realize functionalization on the outer and inner walls more easily, but also show exceptional applications in bioanalysis, catalysis and optic devices. Therefore, researchers have paid more attention to the designing and synthesis of more new multifunctional silica nanotubes.

Silica nanotubes can be fabricated by many methods including excimer laser ablation ( $\mathrm{Yu}$ et al 1998), sol-gel template (Zhang et al 2002), chemical-vapour-deposition (CVD) (Pan et al 2002), and carbothermal reduction (Wu et al 2001). To date, fabrication methods of most silica nanotubes have focused on the use of templates. For example, Mitchell (2002) prepared silica nanotubes within the pores of porous alumina membrane templates

\footnotetext{
*Author for correspondence (yinzhh06@1zu.cn)
}

by a sol-gel coating technique. Yoon (2006) fabricated the silica nanotube with both ends closed from the $\mathrm{CdS}$ nanorod template which was removed with controlled photon energy. Chen et al (2005) synthesized silica nanotubes using zinc oxide nanowires as templates. Wang (2004) and co-workers synthesized porous hollow silica nanotubes by a sol-gel route using needle-like $\mathrm{CaCO}_{3}$ nanoparticles as the novel inorganic templates, which were prepared by a unique high gravity reactive precipitation technology. Obare et al (2001) synthesized hollow silica nanotubes by silica-coated Au nanorods and dissolution of $\mathrm{Au}$ core in $\mathrm{KCN}$. However, these templates are not suitable for fabricating silica nanotubes in large quantities, because there is still a long way to realize the commercial application of these templates mentioned above.

In this work, we report a facile method of the synthesis of porous hollow silica nanotubes using MWCNTs as templates. Typically, for the as-obtained samples, the thickness of the outer walls is about $10 \mathrm{~nm}$ and the inner diameter is completely dependent on the size of the MWCNTs. What's more, the as-obtained silica nanotubes emit a strong violet light, which is different from the emission bands of silica nanotubes prepared by the sol-gel template method (SGTM).

\section{Experimental}

\subsection{Materials}

The MWCNTs with outer diameters of $40-60 \mathrm{~nm}$ were supplied by Shen Zhen. Nanotech. Port. Co. 3-APS was purchased from Shang Hai Yao Hua Chemical Co. Nitric acid, sulfuric acid and ammonia were all purchased from 

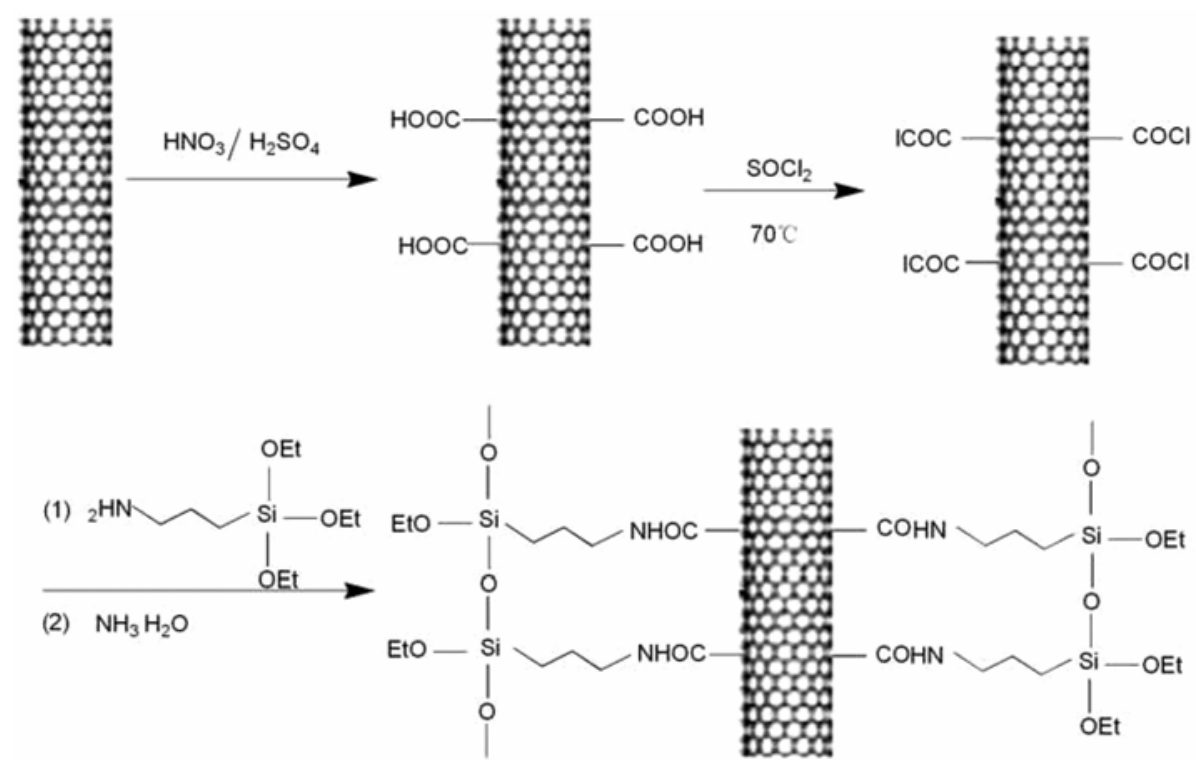

Figure 1. Fabrication procedure of silica coated MWCNTs.

Bei Ying Liang You Chemical Co. Tetrahydrofuran, toluene and thionyl chloride were purchased from Tian Jing Chemical Co. All chemical reagents were of analytical grade and used without further purification.

\subsection{Preparation of silica nanotubes}

In a typical process, the pristine-MWCNTs were added to a 3:1 mixture of concentrated $\mathrm{H}_{2} \mathrm{SO}_{4}$ and $\mathrm{HNO}_{3}$. The suspension was treated in an ultrasonic bath for $15 \mathrm{~min}$ and stirring for $4 \mathrm{~h}$ under reflux. Then, the mixture was centrifuged and washed with distilled water until $\mathrm{pH}$ of the filtrate was 7 . The solid was dried under vacuum at $40^{\circ} \mathrm{C}$ for $24 \mathrm{~h}$ for obtaining MWCNT-COOH.

The acid treated MWCNTs (MWCNT-COOH) were suspended in $\mathrm{SOCl}_{2}$ and the suspension was treated in an ultrasonic bath for $15 \mathrm{~min}$ and stirred at $70^{\circ} \mathrm{C}$ for $24 \mathrm{~h}$. The solid was separated by centrifuge and washed with anhydrous tetrahydrofuran, then dried under vacuum at $40^{\circ} \mathrm{C}$ for $12 \mathrm{~h}$, to produce MWCNT-COCl. The 3-aminopropyltriethoxysilane (3-APS) and anhydrous pyridine were added to a one-neck flask that contained MWCNT$\mathrm{COCl}$ and the mixture was stirred at $120^{\circ} \mathrm{C}$ for $12 \mathrm{~h}$. After the reaction, the solid was centrifuged and washed for several times with toluene to remove residual 3-APS, thus the MWCNT-APS was obtained.

The resulting MWCNT-APS were added to ammonia $(\mathrm{pH}=9)$. The mixture was treated in an ultrasonic bath for $15 \mathrm{~min}$ and stirred at room temperature for $3 \mathrm{~h}$. The solid was centrifuged and washed with distilled water until $\mathrm{pH}$ of the filtrate was 7 . Then the silica coated MWCNTs were obtained. The as-fabricated samples were calcinated at $800^{\circ} \mathrm{C}$ for $4 \mathrm{~h}$ to remove MWCNT template and the final products were obtained. The detailed reaction scheme is shown in figure 1 .

\subsection{Characterization}

Nicolet NEXUS 670 Fourier transform infrared (FTIR) spectrometer, Hitachi 600 transmission electron microscopy (TEM), JEOL JSM-6701 field emission scanning electron microscope (FESEM) and Rigaku D/Max 2400 $\mathrm{X}$-ray diffraction (XRD) analysis were used to characterize the changes in chemical structure of silica coated MWCNTs and chemical structure of silica coated MWCNTs after calcination. F-4500 photoluminescent (PL) spectroscopy was used to characterize the optical properties of silica nanotubes.

\section{Results and discussion}

Figure 2 shows the FTIR spectra of MWCNTs obtained at different possessing steps. The bond at $1710 \mathrm{~cm}^{-1}$ in figure $2 \mathrm{~b}$ is clearly assigned to the stretching mode of carboxyl groups, indicating the presence of $-\mathrm{COOH}$ on the surfaces of MWCNTs. The bands in $1095 \mathrm{~cm}^{-1}$ are assigned to the characteristic absorption peaks of $\mathrm{Si}-\mathrm{O}$ vibration, as shown in figure $2 \mathrm{c}$. The results reveal that 3-APS has grafted onto the MWCNT-COOH. Figure 2d shows the FTIR spectra of the silica coated MWCNTs after calcination. It has strengthened the characteristic absorption peaks of $\mathrm{Si}-\mathrm{O}$ which appear at 1170,1084 , 794 and $466 \mathrm{~cm}^{-1}$. In addition, a broad shoulder band in the $3200-3600 \mathrm{~cm}^{-1}$ and $1630 \mathrm{~cm}^{-1}$ regions also appear in figure $2 \mathrm{~d}$, which can be attributed to the traces of water in the KBr pellet used for the analysis. The result suggests 

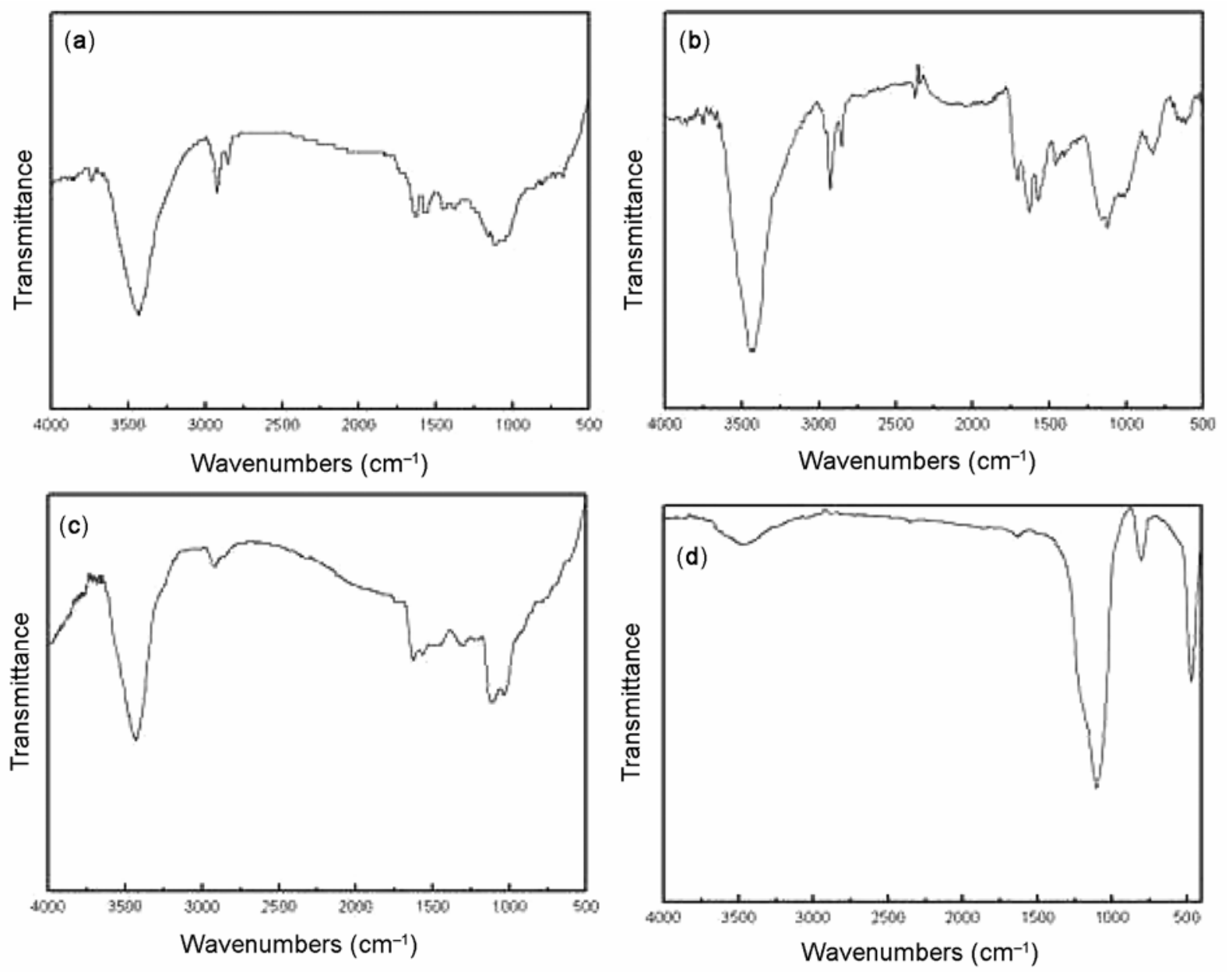

Figure 2. FTIR spectra of different MWCNTs: (a) the raw MWCNTs, (b) the acid treated MWCNTs, (c) silica coated MWCNTs and (d) the silica coated MWCNTs after calcination.

that the core MWCNTs have been removed from the core-shells nanotubes after calcination and that silica nanotubes have been obtained.

The TEM and FESEM images of silica coated MWCNTs without calcination and silica coated MWCNTs after calcination with different magnifications are shown in figure 3. As seen in figure 3a, the MWCNTs after acid treatment was clean and was not covered with any extra phase with some defects on the wall surface. In contrast, figure $3 \mathrm{~b}$ exhibited the silica layer to be uniform, which further confirms that the 3-APS has been grafted onto the surface of MWCNTs successfully. Figures $3 \mathrm{c}$ and d demonstrate the FESEM and TEM images of the silica nanotubes. It can be seen that the silica nanotubes have a smooth surface and average diameter of silica nanotubes is in the range of $50-70 \mathrm{~nm}$. The thickness of the outer walls is about $10 \mathrm{~nm}$ and the inner diameter is completely dependent on the size of MWCNTs.

Figure 4 shows XRD patterns of silica coated MWCNTs after calcination. The broadening of the diffraction peak centred around $23^{\circ}$ confirmed the formation of amor- phous silica. The ED patterns which display a diffusive ring (inset in figure 3d), also suggests that the as-obtained silica nanotubes are amorphous. No diffraction peaks related to MWCNTs are found in the spectrum of the silica nanotubes, which further prove that the MWCNTs cores are completely removed.

The optical properties of the as-obtained silica nanotubes are investigated by PL spectrum at room temperature. Figure 5 presents the photoluminescence (PL) spectrum. The strong violet light emission at $400 \mathrm{~nm}$ is observed under excitation of $250 \mathrm{~nm}$. This result was different from the emission bands of silica nanotubes prepared by the sol-gel template method (SGTM) (Zhang et al 2002). The PL spectra of silica nanotubes prepared by SGTM had maxima at $486 \mathrm{~nm}$ and $539 \mathrm{~nm}$. This phenomenon indicates that the framework of the as-prepared silica nanotubes is inconsistent with that of silica nanotubes prepared by SGTM. According to the previous report (Jang and Yoon 2004), the luminescence characteristic depends on the silica structure, which is affected by the synthetic variables such as the solvent used, its water 


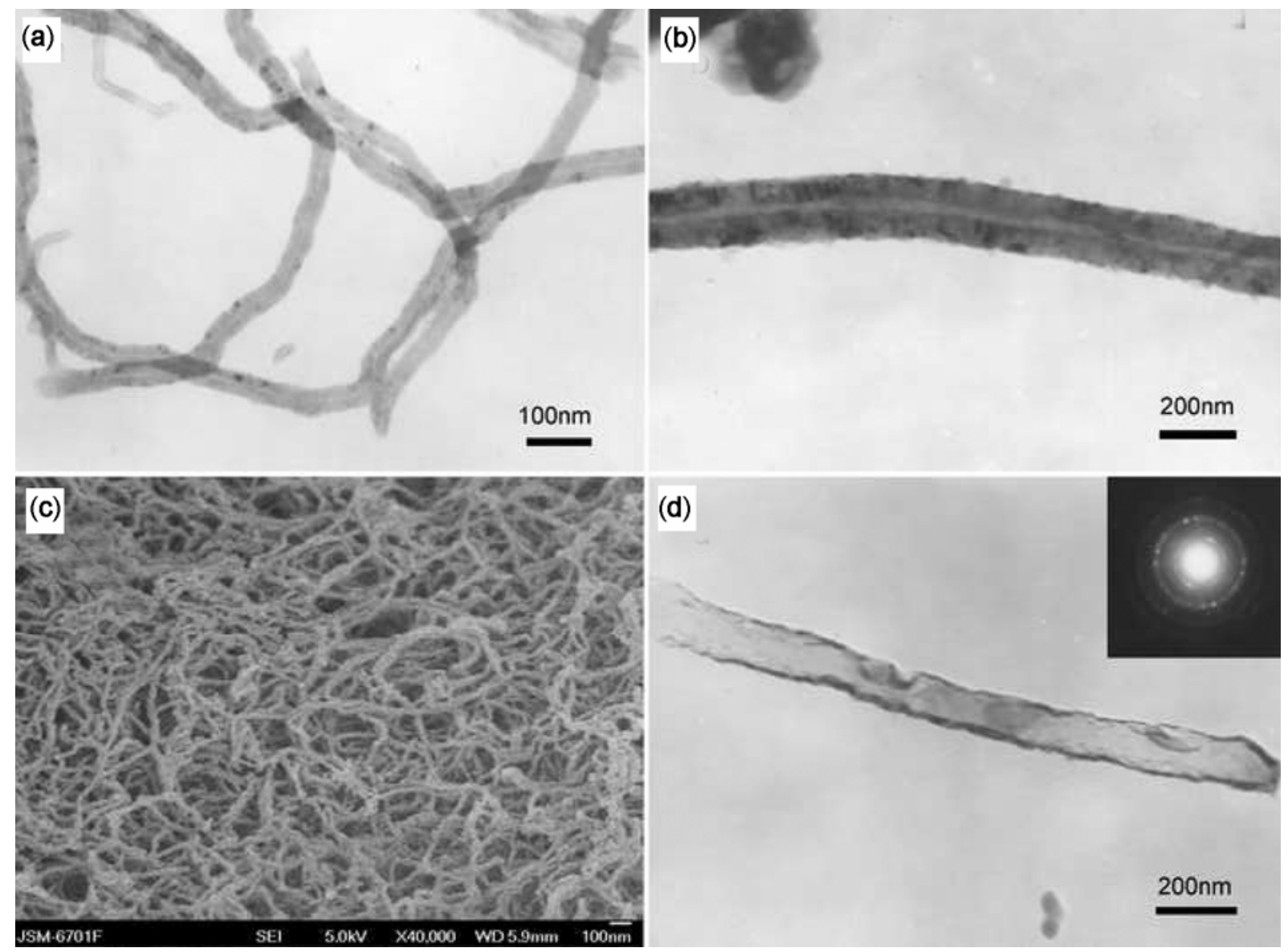

Figure 3. TEM images of the (a) acid treated MWCNTs, (b) MWCNT-APS. FESEM and TEM images and silica coated MWCNTs after calcination $(\mathbf{c}, \mathbf{d})$.

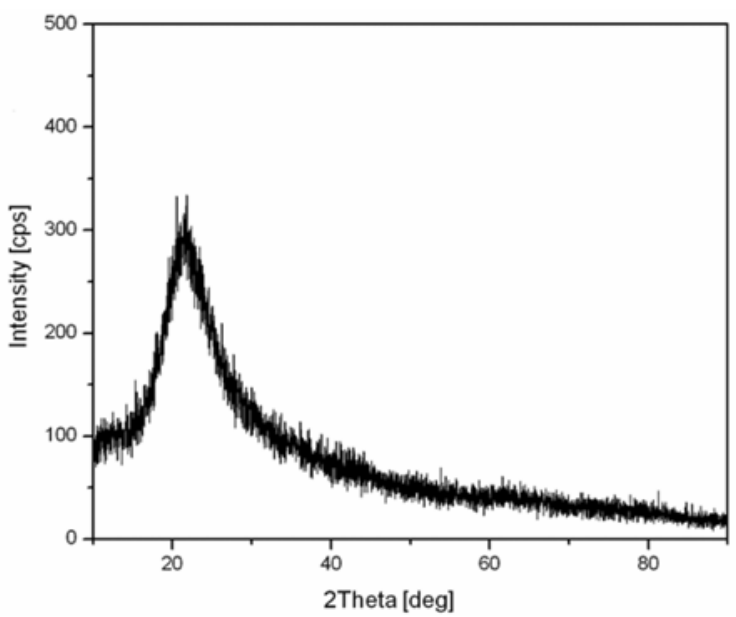

Figure 4. XRD patterns of silica nanotubes.

content, and $\mathrm{pH}$ of the reaction solution. So, we think the noticeable PL spectra of the as-fabricated silica nanotubes can be attributed to their special structure. Nevertheless, the extra nature of the silica nanotubes remains unclear and requires more detailed systematic investigations.

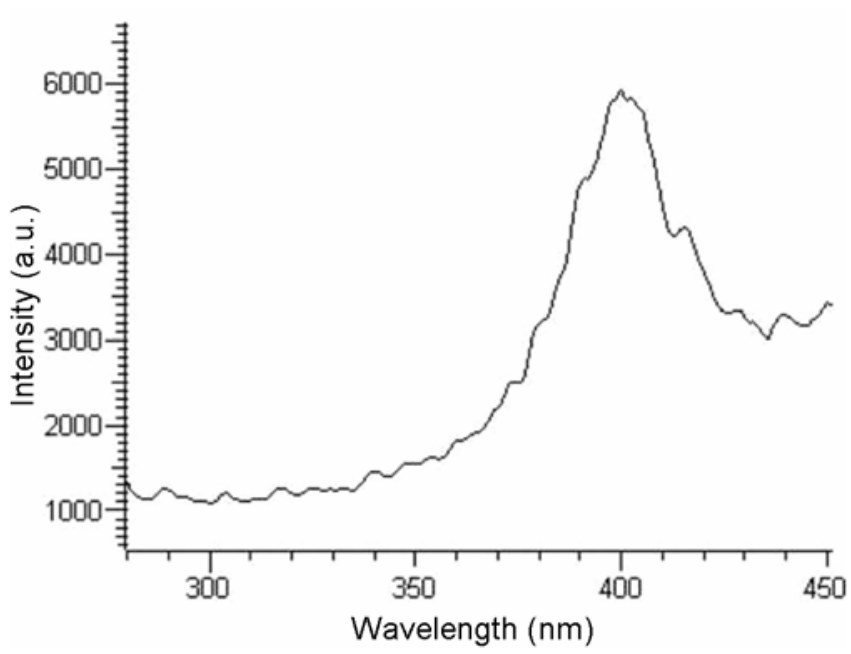

Figure 5. Room temperature PL spectrum of the as-fabricated silica nanotubes.

\section{Conclusions}

In summary, silica nanotubes were synthesized using the multi-walled carbon nanotubes (MWCNTs) as template. 
The outer wall thickness of the silica nanotubes was about $10 \mathrm{~nm}$. The inner diameter is completely dependent on the size of MWCNTs. Additionally, the size control of nanotubes materials may be easily achieved by changing the diameter of MWCNTs template. The strong violet light emission at $400 \mathrm{~nm}$ is observed under excitation of $250 \mathrm{~nm}$. It could provide potential applications in the fields of light localization, lower dimensional waveguides, and scanning near-field optical microscopy.

\section{Acknowledgement}

The authors would like to thank Jin Chuan Group Limited (JNMC) for financial support.

\section{References}

Alivisatos A P 1996 Science 271933

Cheng B and Samulski E T 2001 J. Mater. Chem. 112901

Chen Y, Xue X and Wang T 2005 Nanotechnology 161978

Feldman Y, Wasserman E, Srolovitz D J and Tenne R 1995 Science 26222

Golberg D, Li Y B, Mitome M and Bando Y 2005 Chem. Phys. Lett. 40975

Guo Y G, Hu J S, Liang H P, Wan L J and Bai C L 2005 Adv. Funct. Mater. 15196
Hoyer P 1996 Langmuir 121411

Jang B J and Yoon H 2004 Adv. Mater. 169

Mitchell D T, Lee S B, Trofin L, Li N, Nevaanen T K, Soderlund H and Martin C R 2002 J. Am. Chem. Soc. 12411864

Nakamura H and Matsui Y 1995 J. Am. Chem. Soc. 1172651

Obare S O, Jana N R and Murphy C J 2001 Nano Lett. 1601

Pan Z W, Dai Z R, Ma C and Wang Z L $2002 \mathrm{~J}$. Am. Chem. Soc. 1241817

Schonenberger C et al 1997 J. Phys. Chem. B101 5497

Wang J X, Wen L X, Wang Z H, Wang M, Shao L and Chen J F 2004 Scr. Mater. 511035

Wu X C, Song W H, Wang K Y, Hu T, Zhao B, Sun Y P and Du J J 2001 Chem. Phys. Lett. 33653

Xiang Y, Li Z, Zhang R, Xie Y, Yang J and Wu C 2003 J. Phys. Chem. B107 3697

Ye C H, Meng G W, Jiang Z, Wang Y H, Wang G Z and Zhang L B 2002 J. Am. Chem. Soc. 12415180

Yoon J H, Chae W S, Cho H M, Choi M G and Kim Y R 2006 Mater. Res. Bull. 411657

Yu D P et al 1998 Appl. Phys. Lett. 733076

Zhang J H, Yang X G, Wang D W, Li S D, Xie Y, Xia Y N and Qian Y T 2000 Adv. Mater. 121348

Zhang K F, Liu X, Su Z X and Li H L 2007 Mater. Letts 61 2644

Zhang M, Ciocan E, Bando Y, Wada K, Cheng L L and Pirouz P 2002 Appl. Phys. Lett. 80491

Zhang M, Ciocan E, Bando Y, Wada K, Cheng L L and Pirouz P 2002 Appl. Phys. Lett. 80491

Zollfrank C, Scheel H and Greil P 2007 Adv. Mater. 19984 